\title{
Correspondence between Frame Shrinkage and High-order Nonlinear Diffusion
}

\author{
Qingtang Jiang* \\ Department of Mathematics and Computer Science \\ University of Missouri-St. Louis \\ St. Louis, MO 63121, USA.
}

\begin{abstract}
Nonlinear diffusion filtering and wavelet/frame shrinkage are two popular methods for signal and image denoising. The relationship between these two methods has been studied recently. In this paper we investigate the correspondence between frame shrinkage and nonlinear diffusion.

We show that the frame shrinkage of Ron-Shen's continuous-linear-spline-based tight frame is associated with a fourth-order nonlinear diffusion equation. We derive high-order nonlinear diffusion equations associated with general tight frame shrinkages. These high-order nonlinear diffusion equations are different from the high-order diffusion equations studied in the literature. We also construct two sets of tight frame filter banks which result in the sixth- and eighth-order nonlinear diffusion equations.

The correspondence between frame shrinkage and diffusion filtering is useful to design diffusion-inspired shrinkage functions with competitive performance. On the other hand, the study of such a correspondence leads to a new type of diffusion equations and helps to design frame-inspired diffusivity functions. The denoising results with diffusion-inspired shrinkages provided in this paper are promising.
\end{abstract}

Key words and phrases: Nonlinear diffusion filtering, high-order nonlinear diffusion, signal denoising, undecimated frame filter banks, frame shrinkage, connection between nonlinear diffusion and frame shrinkage

\section{Introduction}

Nonlinear diffusion filtering [26] and wavelet shrinkage (see e.g. [15, 16, 22]) are two powerful methods for signal and image denoising. Correspondence between these two methods has been studied in $[23,31]$. In this paper we investigate the correspondence between frame shrinkage and nonlinear diffusion.

For a given 1-D signal $f$ with a noise, nonlinear diffusion filtering is to obtain $u=u(x, t)$ satisfying the nonlinear diffusion equation

$$
u_{t}=\frac{\partial}{\partial x}\left(g\left(u_{x}^{2}\right) u_{x}\right)
$$

with the initial condition

$$
u(x, 0)=f(x),
$$

*Correspondence author. E-mail: jiangq@umsl.edu, Tel: 1-314-516-6358, Fax: 1-314-516-5400. 
and certain boundary conditions, where $g$ is the diffusivity and $u_{x}$ denotes the first-order partial derivative of $u(x, t)$ with respect to $x$. The diffusivity $g$ is a nonnegative decreasing function controlling the diffusion. The solution $u(x, t)$ of the above nonlinear equation is a denoised version of $f(x)$.

Since the nonlinear diffusion was introduced by Perona and Malik in 1990, a variety of nonlinear diffusion filters have been proposed, see e.g. [6, 36, 13] and the references therein. The fourthorder nonlinear diffusion was proposed in $[38,39]$ to solve the problem that (the second-order) Perona and Malik diffusion and its variants tend to produce blocky effects in image denoising. The fourth-order nonlinear diffusion has also been studied in [21], and high-order diffusion with an edge enhancing functional was proposed in [35]. The theoretical properties of high-order diffusion have been studied in [14]. A 1-D high-order diffusion equation is an equation like

$$
u_{t}=(-1)^{n+1} \frac{\partial^{n}}{\partial x^{n}}\left(g\left(\left(\frac{\partial^{n} u}{\partial x^{n}}\right)^{2}\right) \frac{\partial^{n} u}{\partial x^{n}}\right),
$$

for an integer $n \geq 2$.

The discretization of (1.1) could be given as follows. Let $h$ denote the spatial step size and let $\tau$ be the time step size. Denote

$$
u_{k}^{0}=f(k h), \quad k \in \mathbb{Z} .
$$

We use $u_{k}^{j}, j \geq 1$ to denote the (approximation) value of the solution $u(x, t)$ at $(k h, j \tau)$. Thus $u^{j}$ is the approximation solution at time $j \tau$. With the facts that $\left(u_{k}^{j+1}-u_{k}^{j}\right) / \tau$ approximates $u_{t}$ at $(k h, j \tau)$ and $\left(u_{k+1}^{j}-u_{k}^{j}\right) / h$ approximates $u_{x}$ at $(k h, j \tau)$, equation (1.1) can be discretized as

$$
u_{k}^{j+1}=u_{k}^{j}+\frac{\tau}{h^{2}} g\left(\left(\frac{u_{k+1}^{j}-u_{k}^{j}}{h}\right)^{2}\right)\left(u_{k+1}^{j}-u_{k}^{j}\right)-\frac{\tau}{h^{2}} g\left(\left(\frac{u_{k}^{j}-u_{k-1}^{j}}{h}\right)^{2}\right)\left(u_{k}^{j}-u_{k-1}^{j}\right),
$$

for $j=0,1, \cdots$.

Wavelets have been successfully used in signal and image processing $[15,16,22,33]$. In particular, the undecimated wavelet transform (UWT) (also called the shift-invariant wavelet transform) based denoising [11] has been used widely for signal and image denoising. Let $\{p, q\}$ be a wavelet filter bank. For a given signal $\left\{c_{k}\right\}_{k}$, the UWT-based denoising consists of the analysis step:

$$
L_{n}=\frac{1}{\sqrt{2}} \sum_{k \in \mathbb{Z}} p_{k} c_{k+n}, H_{n}=\frac{1}{\sqrt{2}} \sum_{k \in \mathbb{Z}} q_{k} c_{k+n}
$$

and the synthesis step:

$$
u_{k}=\frac{\sqrt{2}}{4} \sum_{n \in \mathbb{Z}} p_{n} L_{k-n}+\frac{\sqrt{2}}{4} \sum_{n \in \mathbb{Z}} q_{n} S_{\theta}\left(H_{k-n}\right),
$$

where $S_{\theta}$ is the shrinkage function, depending a parameter $\theta$ (or several parameters). With a suitable shrinkage function (for example, the hard or soft shrinkage function), $\left\{u_{k}\right\}_{k}$ is the denoised signal of the original signal $\left\{c_{k}\right\}_{k}$ with noise.

It was shown in [23] that when $p, q$ are the Haar filter pair, namely, $p_{0}=p_{1}=1, q_{0}=1, q_{1}=$ $-1, p_{k}=0, q_{k}=0, k \neq 0,1$, then $u_{k}$ in (1.5) is $u_{k}^{1}$ in (1.3) provided that shrinkage function $S_{\theta}$ and the diffusivity $g$ satisfy

$$
S_{\theta}(x)=x\left(1-\frac{4 \tau}{h^{2}} g\left(\frac{2 x^{2}}{h^{2}}\right)\right)
$$


where $\theta$ is the parameter with the diffusivity $g$. Namely, iterated Haar wavelet shrinking and the 2nd-order diffusion filtering result in the same signal. This relationship reveals the connection between nonlinear diffusion filtering and wavelet shrinkage and hence, it opens the gate of exchanging ideas between these two fields. In particular, the connection helps to choose shrinkage functions from diffusivity functions, and vice versa. Refer to $[23,31,24]$ for the detailed discussion on the importance of the relationship. The reader is referred to $[1,10]$ for the relationship between PDE diffusion and the bilateral filter, another popular method for image denoising.

Recently wavelet frames have been successfully used in noise removal [30], image recovery $[7,8]$, image inpainting/restoration [3, 4, 5], signal classification [9] and medical image analysis [18, 25]. Compared with wavelet systems, the elements in a frame system may be linearly dependent; namely, frames can be redundant. The property of redundancy not only provides a flexibility for the construction of framelets with desirable properties, but also provides high sparsity of frame transform coefficients. Such sparsity is a key property for many applications. In addition, frames work better in a noisy environment [9]. It is very natural to ask whether there is a correspondence between frame shrinkage functions and the nonlinear diffusivity functions of some diffusion equations. In this paper we show that the undecimated frame shrinking corresponds to a high-order nonlinear diffusion such as

$$
u_{t}=\frac{\partial}{\partial x}\left(g_{1}\left(u_{x}^{2}\right) u_{x}\right)-\frac{\partial^{2}}{\partial x^{2}}\left(g_{2}\left(u_{x x}^{2}\right) u_{x x}\right)
$$

with $f$ as initial condition:

$$
u(x, 0)=f(x),
$$

where $u_{x x}$ denotes the second-order partial derivative of $u(x, t)$ with respect to $x$. Observe that the high-order diffusion equation corresponding to a frame shrinkage is different from the high-order diffusion equations like (1.2) considered in [38, 39, 21, 14].

The rest of the paper is organized as follows. In Section 2, we show how Ron-Shen's continuouslinear-spline-based tight frame shrinkage corresponds to the diffusion equation given in (1.7). In Section 3, we consider the general case. We show how the vanishing moment of a highpass filter $q^{(\ell)}$ is related to the order of a nonlinear diffusion equation and derive high-order nonlinear diffusion equations associated with general tight frame shrinkages. In Section 4, we construct two sets of tight frame filter banks which result in the 6th-order and 8th-order diffusion equations. In Section 5 , we provide some experiment results. We draw the conclusion in Section 6 .

\section{Fourth-order diffusion and tight frame shrinkage correspon- dence}

In this section we show how Ron-Shen's continuous-linear-spline-based tight frame shrinkage corresponds to a 4th-order nonlinear diffusion equation.

\subsection{Ron-Shen's tight frame shrinkage}

For a sequence $\left\{p_{k}\right\}_{k \in \mathbb{Z}}$ of real numbers, we use $p(\omega)$ to denote its symbol (also called filter here):

$$
p(\omega)=\frac{1}{2} \sum_{k \in \mathbb{Z}} p_{k} e^{-i k \omega} .
$$


Let $\left\{p, q^{(1)}, \cdots, q^{(L)}\right\}$ and $\left\{\widetilde{p}, \widetilde{q}^{(1)}, \cdots, \widetilde{q}^{(L)}\right\}$ be a pair of FIR frame filter banks. Assume that they are biorthogonal, namely,

$$
\begin{aligned}
& \overline{p(\omega)} \widetilde{p}(\omega)+\sum_{\ell=1}^{L} \overline{q^{(\ell)}(\omega)} \widetilde{q}^{(\ell)}(\omega)=1, \\
& \overline{p(\omega)} \widetilde{p}(\omega+\pi)+\sum_{\ell=1}^{L} \overline{q^{(\ell)}(\omega)} \widetilde{q}^{(\ell)}(\omega+\pi)=0 .
\end{aligned}
$$

If a filter bank $\left\{p, q^{(1)}, \cdots, q^{(L)}\right\}$ satisfies $(2.1)$ and $(2.2)$ with $\widetilde{p}=p, \widetilde{q}^{(\ell)}=q^{(\ell)}, 1 \leq \ell \leq L$, then it is called a tight frame filter bank. It was shown in [28] that if compactly supported scaling functions $\phi, \widetilde{\phi}$ corresponding to lowpass filters $p, \widetilde{p}$ are in $L^{2}(\mathbb{R})$ with $\int_{\mathbb{R}} \phi(x) d x \neq 0, \int_{\mathbb{R}} \widetilde{\phi}(x) d x \neq 0$, and $p(0)=\widetilde{p}(0)=1, p(\pi)=\widetilde{p}(\pi)=q^{(\ell)}(0)=\widetilde{q}^{(\ell)}(0)=0,1 \leq \ell \leq L$, then biorthogonal frame filter banks generate wavelet bi-frames (also called dual wavelet frames) of $L^{2}(\mathbb{R})$.

Let $\left\{c_{k}\right\}_{k}$ be the initial data. The undecimated frame transform (UFT) based denoising consists of the analysis step:

$$
L_{n}=\frac{1}{\sqrt{2}} \sum_{k \in \mathbb{Z}} p_{k} c_{k+n}, H_{n}^{(\ell)}=\frac{1}{\sqrt{2}} \sum_{k \in \mathbb{Z}} q_{k}^{(\ell)} c_{k+n}, \quad n \in \mathbb{Z}, \ell=1, \cdots, L,
$$

and the synthesis step:

$$
u_{k}=\frac{\sqrt{2}}{4} \sum_{n \in \mathbb{Z}} \widetilde{p}_{n} L_{k-n}+\frac{\sqrt{2}}{4} \sum_{\ell=1}^{L} \sum_{n \in \mathbb{Z}} \widetilde{q}_{n}^{(\ell)} S_{\theta_{\ell}}^{\ell}\left(H_{k-n}^{(\ell)}\right),
$$

where $S_{\theta_{\ell}}^{\ell}, 1 \leq \ell \leq L$ are the shrinkage functions, depending on parameters $\theta_{\ell}$. One can easily verify that when $S_{\theta_{\ell}}^{\ell}(x)=x, 1 \leq \ell \leq L, u_{k}$ is $c_{k}$ provided that $\left\{p, q^{(1)}, \cdots, q^{(L)}\right\}$ and $\left\{\widetilde{p}, \widetilde{q}^{(1)}, \cdots, \widetilde{q}^{(L)}\right\}$ satisfy (2.1). Namely, in this case the synthesis step recovers the original signal.

In this section we consider a particular tight frame filter bank from [27]. The corresponding scaling function is the continuous linear spline function (hat function) supported on $[-1,1]$. In this paper we call this filter bank Ron-Shen's tight frame filter bank. The nonzero coefficients of the filters are

$$
p_{0}=1, p_{1}=p_{-1}=\frac{1}{2}, q_{0}^{(1)}=0, q_{-1}^{(1)}=\frac{\sqrt{2}}{2}, q_{1}^{(1)}=-\frac{\sqrt{2}}{2}, q_{0}^{(2)}=1, q_{-1}^{(2)}=q_{1}^{(2)}=-\frac{1}{2} .
$$

With this tight frame filter bank, $L_{n}, H_{n}^{(1)}, H_{n}^{(2)}$ defined by (2.3) are

$$
L_{n}=\frac{\sqrt{2}}{4}\left(c_{n-1}+2 c_{n}+c_{n+1}\right), \quad H_{n}^{(1)}=\frac{1}{2}\left(c_{n-1}-c_{n+1}\right), \quad H_{n}^{(2)}=\frac{\sqrt{2}}{4}\left(2 c_{n}-c_{n-1}-c_{n+1}\right) .
$$

Let $S_{\theta}^{1}$ and $S_{\sigma}^{2}$ denote the frame shrinkage operators applied to the first and second highpass outputs $\left\{H_{n}^{(1)}\right\}_{n}$ and $\left\{H_{n}^{(2)}\right\}_{n}$ respectively. Then the denoised signal $u_{k}$ after the synthesis step $(2.4)$ is

$$
\begin{gathered}
u_{k}=\frac{\sqrt{2}}{4}\left(L_{k}+\frac{1}{2} L_{k-1}+\frac{1}{2} L_{k+1}\right)+\frac{\sqrt{2}}{4} \frac{\sqrt{2}}{2}\left(S_{\theta}^{1}\left(H_{k+1}^{(1)}\right)-S_{\theta}^{1}\left(H_{k-1}^{(1)}\right)\right) \\
+\frac{\sqrt{2}}{4}\left(S_{\sigma}^{2}\left(H_{k}^{(2)}\right)-\frac{1}{2} S_{\sigma}^{2}\left(H_{k+1}^{(2)}\right)-\frac{1}{2} S_{\sigma}^{2}\left(H_{k-1}^{(2)}\right)\right) .
\end{gathered}
$$


With $L_{k}, H_{k}^{(1)}$ and $H_{k}^{(2)}$ given by $(2.6), u_{k}$ can be written as

$$
\begin{aligned}
u_{k}= & \frac{1}{16}\left(c_{k-2}+4 c_{k-1}+6 c_{k}+4 c_{k+1}+c_{k+2}\right)+\frac{1}{4} S_{\theta}^{1}\left(\frac{c_{k}-c_{k+2}}{2}\right)-\frac{1}{4} S_{\theta}^{1}\left(\frac{c_{k-2}-c_{k}}{2}\right) \\
+ & \frac{\sqrt{2}}{4} S_{\sigma}^{2}\left(\frac{\sqrt{2}}{4}\left\{2 c_{k}-c_{k-1}-c_{k+1}\right\}\right)-\frac{\sqrt{2}}{8} S_{\sigma}^{2}\left(\frac{\sqrt{2}}{4}\left\{2 c_{k-1}-c_{k-2}-c_{k}\right\}\right) \\
& \quad-\frac{\sqrt{2}}{8} S_{\sigma}^{2}\left(\frac{\sqrt{2}}{4}\left\{2 c_{k+1}-c_{k}-c_{k+2}\right\}\right) .
\end{aligned}
$$

With suitable shrinkage functions $S_{\theta}^{1}$ and $S_{\sigma}^{2}, u_{k}$ is the denoised signal after one step of frame denoising process of the original $c_{k}$ with noise. We can apply the above denoising process to $u_{k}$ to get further denoised signal. In fact we can apply the frame shrinkage process repeatedly to the denoised signal to get further denoised signal. We call this process the iterated frame denoising process. In the next subsection we show that the output after iterated denoising process with Ron-Shen's tight frame filter bank is the same signal resulted by the nonlinear diffusion of a 4th-order diffusion equation.

\subsection{Fourth-order nonlinear diffusion equation}

We consider nonlinear diffusion equation (1.7) for $u=(x, t)$ with $u(x, 0)=f(x)$. To discretize the diffusion equation (1.7), we recall two formulas to approximate derivatives of a function. For a function $L(x)$ on $\mathbb{R}$ and $\varepsilon>0$, we have that (see e.g. [2])

$$
L^{\prime}\left(x_{0}\right)=\frac{1}{2 \varepsilon}\left(L\left(x_{0}+\varepsilon\right)-L\left(x_{0}-\varepsilon\right)\right)-\frac{\varepsilon^{2}}{6} L^{(3)}\left(\xi_{1}\right),
$$

provided that $L \in C^{3}\left[x_{0}-\varepsilon, x_{0}+\varepsilon\right]$, where $\xi_{1} \in\left[x_{0}-\varepsilon, x_{0}+\varepsilon\right]$; and that

$$
L^{\prime \prime}\left(x_{0}\right)=\frac{1}{\varepsilon^{2}}\left(L\left(x_{0}-\varepsilon\right)-2 L\left(x_{0}\right)+L\left(x_{0}+\varepsilon\right)\right)-\frac{\varepsilon^{2}}{12} L^{(4)}\left(\xi_{2}\right),
$$

provided that $L \in C^{4}\left[x_{0}-\varepsilon, x_{0}+\varepsilon\right]$, where $\xi_{2} \in\left[x_{0}-\varepsilon, x_{0}+\varepsilon\right]$.

Next we discretize (1.7) by using (2.8) and (2.9) to approximate the first- and second-order partial derivatives with respect to the variable $x$ in (1.7). Recall that $h$ and $\tau$ denote the spatial step size and the time step size respectively. As in Section 1 , we use $u^{j}$ to denote the approximation solution of (1.7) at time $j \tau$, and $u_{k}^{j}$ to denote the approximation value of the solution at $(k h, j \tau)$. Thus $\left(u_{k}^{j+1}-u_{k}^{j}\right) / \tau$ is the approximation of $u_{t}$ at $(k h, j \tau)$. We use (2.8) with $\varepsilon=h$ to approximate the first-order partial derivative $u_{x}$ of $u(x, t)$ and the first-order partial derivative of $g_{1}\left(u_{x}^{2}\right) u_{x}$ in the first term on the right-hand side of (1.7), while we use (2.9) with $\varepsilon=h$ to approximate $u_{x x}$ and the second-order partial derivative with respect to $x$ of $g_{2}\left(u_{x x}^{2}\right) u_{x x}$ in the second term on the 
right-hand side of (1.7). Then we have

$$
\begin{aligned}
& \frac{u_{k}^{j+1}-u_{k}^{j}}{\tau}=\frac{1}{2 h}\left\{g_{1}\left(\left(\frac{u_{k+2}^{j}-u_{k}^{j}}{2 h}\right)^{2}\right) \frac{u_{k+2}^{j}-u_{k}^{j}}{2 h}-g_{1}\left(\left(\frac{u_{k}^{j}-u_{k-2}^{j}}{2 h}\right)^{2}\right) \frac{u_{k}^{j}-u_{k-2}^{j}}{2 h}\right\} \\
&-\frac{1}{h^{2}}\left\{g_{2}\left(\left(\frac{u_{k-2}^{j}-2 u_{k-1}^{j}+u_{k}^{j}}{h^{2}}\right)^{2}\right) \frac{u_{k-2}^{j}-2 u_{k-1}^{j}+u_{k}^{j}}{h^{2}}\right. \\
&-2 g_{2}\left(\left(\frac{u_{k-1}^{j}-2 u_{k}^{j}+u_{k+1}^{j}}{h^{2}}\right)^{2}\right) \frac{u_{k-1}^{j}-2 u_{k}^{j}+u_{k+1}^{j}}{h^{2}} \\
&\left.+g_{2}\left(\left(\frac{u_{k}^{j}-2 u_{k+1}^{j}+u_{k+2}^{j}}{h^{2}}\right)^{2}\right) \frac{u_{k}^{j}-2 u_{k+1}^{j}+u_{k+2}^{j}}{h^{2}}\right\} .
\end{aligned}
$$

Thus, we get

$$
\begin{aligned}
u_{k}^{j+1}=u_{k}^{j} & +\frac{\tau}{4 h^{2}} g_{1}\left(\left(u_{k+2}^{j}-u_{k}^{j}\right)^{2} /\left(4 h^{2}\right)\right)\left(u_{k+2}^{j}-u_{k}^{j}\right) \\
& -\frac{\tau}{4 h^{2}} g_{1}\left(\left(u_{k}^{j}-u_{k-2}^{j}\right)^{2} /\left(4 h^{2}\right)\right)\left(u_{k}^{j}-u_{k-2}^{j}\right) \\
& -\frac{\tau}{h^{4}} g_{2}\left(\left(u_{k-2}^{j}-2 u_{k-1}^{j}+u_{k}^{j}\right)^{2} / h^{4}\right)\left(u_{k-2}^{j}-2 u_{k-1}^{j}+u_{k}^{j}\right) \\
& +\frac{2 \tau}{h^{4}} g_{2}\left(\left(u_{k-1}^{j}-2 u_{k}^{j}+u_{k+1}^{j}\right)^{2} / h^{4}\right)\left(u_{k-1}^{j}-2 u_{k}^{j}+u_{k+1}^{j}\right) \\
& -\frac{\tau}{h^{4}} g_{2}\left(\left(u_{k}^{j}-2 u_{k+1}^{j}+u_{k+2}^{j}\right)^{2} / h^{4}\right)\left(u_{k}^{j}-2 u_{k+1}^{j}+u_{k+2}^{j}\right) .
\end{aligned}
$$

Next, we obtain that $u_{k}$ in (2.7) after 1-step frame shrinkage is $u_{k}^{1}$ after 1-step diffusing if $S_{\theta}^{1}$ and $S_{\sigma}^{2}$ are related to $g_{1}(x)$ and $g_{2}(x)$ respectively as given in the next theorem.

Theorem 1. Let $u_{k}$ in (2.7) be the resulting signal after 1-step frame shrinking with input $c_{k}=$ $f(k h), k \in \mathbb{Z}$ and $u_{k}^{1}$ in (2.10) be the signal after 1-step diffusing with the initial input $u_{k}^{0}=$ $f(k h), k \in \mathbb{Z}$. If

$$
S_{\theta}^{1}(x)=x\left(1-\frac{2 \tau}{h^{2}} g_{1}\left(\frac{x^{2}}{h^{2}}\right)\right), \quad S_{\sigma}^{2}(x)=x\left(1-\frac{16 \tau}{h^{4}} g_{2}\left(\frac{8 x^{2}}{h^{4}}\right)\right),
$$

then $u_{k}=u_{k}^{1}$ for all $k$.

Proof. With $u_{k}^{0}=c_{k}, u_{k}^{1}$ in (2.10) after 1 step diffusion is

$$
\begin{aligned}
u_{k}^{1}=c_{k}+ & \frac{\tau}{4 h^{2}} g_{1}\left(\left(c_{k+2}-c_{k}\right)^{2} /\left(4 h^{2}\right)\right)\left(c_{k+2}-c_{k}\right) \\
& -\frac{\tau}{4 h^{2}} g_{1}\left(\left(c_{k}-c_{k-2}\right)^{2} /\left(4 h^{2}\right)\right)\left(c_{k}-c_{k-2}\right) \\
& -\frac{\tau}{h^{4}} g_{2}\left(\left(c_{k-2}-2 c_{k-1}+c_{k}\right)^{2} / h^{4}\right)\left(c_{k-2}-2 c_{k-1}+c_{k}\right) \\
& +\frac{2 \tau}{h^{4}} g_{2}\left(\left(c_{k-1}-2 c_{k}+c_{k+1}\right)^{2} / h^{4}\right)\left(c_{k-1}-2 c_{k}+c_{k+1}\right) \\
& -\frac{\tau}{h^{4}} g_{2}\left(\left(c_{k}-2 c_{k+1}+c_{k+2}\right)^{2} / h^{4}\right)\left(c_{k}-2 c_{k+1}+c_{k+2}\right) .
\end{aligned}
$$


Write $c_{k}$ as

$$
\begin{aligned}
c_{k}= & \frac{1}{16}\left(c_{k-2}+4 c_{k-1}+6 c_{k}+4 c_{k+1}+c_{k+2}\right)-\frac{1}{8}\left(c_{k+2}-c_{k}\right)+\frac{1}{8}\left(c_{k}-c_{k-2}\right) \\
& +\frac{1}{16}\left(c_{k-2}-2 c_{k-1}+c_{k}\right)-\frac{1}{8}\left(c_{k-1}-2 c_{k}+c_{k+1}\right)+\frac{1}{16}\left(c_{k}-2 c_{k+1}+c_{k+2}\right) .
\end{aligned}
$$

Then we have that

$$
\begin{aligned}
u_{k}^{1}=\frac{1}{16} & \left(c_{k-2}+4 c_{k-1}+6 c_{k}+4 c_{k+1}+c_{k+2}\right) \\
& +\left(\frac{\tau}{4 h^{2}} g_{1}\left(\left(c_{k+2}-c_{k}\right)^{2} /\left(4 h^{2}\right)\right)-\frac{1}{8}\right)\left(c_{k+2}-c_{k}\right) \\
& -\left(\frac{\tau}{4 h^{2}} g_{1}\left(\left(c_{k}-c_{k-2}\right)^{2} /\left(4 h^{2}\right)\right)-\frac{1}{8}\right)\left(c_{k}-c_{k-2}\right) \\
& +\left(\frac{1}{16}-\frac{\tau}{h^{4}} g_{2}\left(\left(c_{k-2}-2 c_{k-1}+c_{k}\right)^{2} / h^{4}\right)\right)\left(c_{k-2}-2 c_{k-1}+c_{k}\right) \\
& -2\left(\frac{1}{16}-\frac{\tau}{h^{4}} g_{2}\left(\left(c_{k-1}-2 c_{k}+c_{k+1}\right)^{2} / h^{4}\right)\right)\left(c_{k-1}-2 c_{k}+c_{k+1}\right) \\
& +\left(\frac{1}{16}-\frac{\tau}{h^{4}} g_{2}\left(\left(c_{k}-2 c_{k+1}+c_{k+2}\right)^{2} / h^{4}\right)\right)\left(c_{k}-2 c_{k+1}+c_{k+2}\right) .
\end{aligned}
$$

Comparing (2.7) with (2.12), we obtain that $u_{k}$ in (2.7) after 1-step frame shrinking is $u_{k}^{1}$ after 1-step diffusing if $S_{\theta}^{1}, S_{\sigma}^{2}, g_{1}(x)$ and $g_{2}(x)$ satisfy (2.11).

From Theorem 1, we immediately have the following corollary.

Corollary 1. With diffusivity functions $g_{1}(x), g_{2}(x)$ and shrinkage functions $S_{\theta}^{1}, S_{\sigma}^{2}$ satisfying (2.11), iterated frame shrinking with Ron-Shen's tight frame filer bank and nonlinear diffusing with (1.7) result in the same signal.

The correspondence (2.11) between frame shrinkage and diffusion filtering is useful to design diffusion-inspired shrinkage functions for frame signal denoising. On the other hand, this correspondence is useful to design frame-inspired diffusivity functions. In the following we give the corresponding shrinkage functions $S_{\theta}^{1}, S_{\sigma}^{2}$ when diffusivity functions $g_{1}, g_{2}$ are the Perona-Malik diffusivity and Weickert diffusivity functions, and provide the associated diffusivity functions $g_{1}, g_{2}$ when $S_{\theta}^{1}, S_{\sigma}^{2}$ are the hard shrinkage and soft shrinkage functions. The reader is referred to $[23,31,24]$ for more diffusivity and shrinkage functions.

Assume the spatial step size $h=1$. Corresponding to the Perona-Malik diffusivity [26]

$$
g\left(x^{2}\right)=\frac{c}{1+(x / \lambda)^{2}},
$$

where $c$ is a constant, shrinkage functions $S_{\theta}^{1}, S_{\sigma}^{2}$ are

$$
S_{\theta}^{1}(x)=x\left(1-\frac{2 \tau c_{1}}{1+(x / \theta)^{2}}\right), S_{\sigma}^{2}(x)=x\left(1-\frac{16 \tau c_{2}}{1+(2 \sqrt{2} x / \sigma)^{2}}\right)
$$

while corresponding to the TV diffusivity [29], shrinkage functions $S_{\theta}^{1}, S_{\sigma}^{2}$ are

$$
S_{\theta}^{1}(x)=x-2 \tau \operatorname{sgn}(x), S_{\sigma}^{2}(x)=x-4 \sqrt{2} \tau \operatorname{sgn}(x) .
$$


If $g_{1}, g_{2}$ are the Weickert diffusivity [36] given by

$$
g\left(x^{2}\right)= \begin{cases}1, & \text { if } x=0, \\ 1-\exp \left(-3.31488 \lambda^{8} / x^{8}\right), & \text { if } x \neq 0,\end{cases}
$$

then the corresponding shrinkage functions $S_{\theta}^{1}, S_{\sigma}^{2}$ are

$$
\begin{aligned}
& S_{\theta}^{1}(x)= \begin{cases}0, & \text { if } x=0, \\
x\left(1-2 \tau+2 \tau \exp \left(-3.31488 \theta^{8} / x^{8}\right)\right), & \text { if } x \neq 0,\end{cases} \\
& S_{\sigma}^{2}(x)= \begin{cases}0, & \text { if } x=0, \\
x\left(1-16 \tau+16 \tau \exp \left(-3.31488 \sigma^{8} /(2 \sqrt{2} x)^{8}\right)\right), & \text { if } x \neq 0 .\end{cases}
\end{aligned}
$$

If $S_{\theta}^{1}, S_{\sigma}^{2}$ are the hard shrinkage functions [15, 22]:

$$
S_{\theta}^{1}(x)=\left\{\begin{array}{ll}
0, & \text { if }|x| \leq \theta, \\
x, & \text { if }|x|>\theta,
\end{array} \quad S_{\sigma}^{2}(x)= \begin{cases}0, & \text { if }|x| \leq \sigma \\
x, & \text { if }|x|>\sigma\end{cases}\right.
$$

then the corresponding diffusivity functions $g_{1}, g_{2}$ are

$$
g_{1}\left(x^{2}\right)=\left\{\begin{array}{ll}
\frac{1}{2 \tau}, & \text { if }|x| \leq \theta, \\
0, & \text { if }|x|>\theta,
\end{array} \quad g_{2}\left(x^{2}\right)= \begin{cases}\frac{1}{16 \tau}, & \text { if }|x| \leq 2 \sqrt{2} \sigma, \\
0, & \text { if }|x|>2 \sqrt{2} \sigma .\end{cases}\right.
$$

When $S_{\theta}^{1}, S_{\sigma}^{2}$ are the soft shrinkage functions [16]:

$$
S_{\theta}^{1}(x)=\left\{\begin{array}{ll}
0, & \text { if }|x| \leq \theta, \\
x-\theta \operatorname{sgn}(x), & \text { if }|x|>\theta,
\end{array} \quad S_{\sigma}^{2}(x)= \begin{cases}0, & \text { if }|x| \leq \sigma \\
x-\theta \operatorname{sgn}(x), & \text { if }|x|>\sigma\end{cases}\right.
$$

the corresponding diffusivity functions $g_{1}, g_{2}$ are

$$
g_{1}\left(x^{2}\right)=\left\{\begin{array}{ll}
\frac{1}{2 \tau}, & \text { if }|x| \leq \theta, \\
\frac{\theta}{2 \tau|x|}, & \text { if }|x|>\theta,
\end{array} \quad g_{2}\left(x^{2}\right)= \begin{cases}\frac{1}{16 \tau}, & \text { if }|x| \leq 2 \sqrt{2} \sigma, \\
\frac{\sigma}{4 \sqrt{2} \tau|x|}, & \text { if }|x|>2 \sqrt{2} \sigma .\end{cases}\right.
$$

Here we should point out that the diffusivity functions $g_{1}, g_{2}$ in either (2.17) or (2.18) are not differentiable. Thus their derivatives in the original equation (1.7) should be understood as the differences in (2.10), a discretized version of (1.7).

\subsection{Minimizer of energy functional and Euler-Lagrange equations}

In this subsection we show that the nonlinear diffusion equation (1.7) is related to the EulerLagrange equation of a variational functional.

Let

$$
F\left(u, u^{\prime}, u^{\prime \prime}\right)=(u-f)^{2}+\alpha \Psi\left(\left(u^{\prime}\right)^{2}\right)+\alpha \Phi\left(\left(u^{\prime \prime}\right)^{2}\right),
$$

where $u^{\prime}, u^{\prime \prime}$ denote the first- and second-order derivatives of $u(x)$. Consider the energy functional

$$
E\left(u, u^{\prime}, u^{\prime \prime}\right)=\int_{a}^{b} F\left(u, u^{\prime}, u^{\prime \prime}\right) d x
$$

for $\alpha>0$. A necessary condition for $E$ to gain the minimum is that $u$ satisfies the Euler-Lagrange equation (see [32] at p. 245)

$$
\frac{\partial F}{\partial u}-\frac{d}{d x}\left(\frac{\partial F}{\partial u^{\prime}}\right)+\frac{d^{2}}{d x^{2}}\left(\frac{\partial F}{\partial u^{\prime \prime}}\right)=0 .
$$


With

$$
\frac{\partial F}{\partial x}=2(u-f), \frac{\partial F}{\partial u^{\prime}}=2 \alpha \Psi^{\prime}\left(\left(u^{\prime}\right)^{2}\right) u^{\prime}, \frac{\partial F}{\partial u^{\prime \prime}}=2 \alpha \Phi^{\prime}\left(\left(u^{\prime \prime}\right)^{2}\right) u^{\prime \prime},
$$

we know $(2.19)$ is

$$
2(u-f)-2 \alpha \frac{d}{d x}\left(\Psi^{\prime}\left(\left(u^{\prime}\right)^{2}\right) u^{\prime}\right)+2 \alpha \frac{d^{2}}{d x^{2}}\left(\Phi^{\prime}\left(\left(u^{\prime \prime}\right)^{2}\right) u^{\prime \prime}\right)=0 .
$$

Denote $g_{1}(x)=\Psi^{\prime}(x), g_{2}(x)=\Phi^{\prime}(x)$. Then the above equation can be written as

$$
\frac{u-f}{\alpha}=\frac{d}{d x}\left(g_{1}\left(\left(u^{\prime}\right)^{2}\right) u^{\prime}\right)-\frac{d^{2}}{d x^{2}}\left(g_{2}\left(\left(u^{\prime \prime}\right)^{2}\right) u^{\prime \prime}\right) .
$$

By introducing an artificial time variable $t$ to $u(x)$ and letting $u(x, 0)=f(x)$, the left-hand side of equation (2.20) can be understood as the discretization to the time variable $t$ of $\frac{\partial}{\partial t} u(x, t)$ with step size $\alpha$, and equation (2.20) is a time discretization of the nonlinear diffusion equation (1.7). The reader is referred to [14] for more detailed discussions on the relationship between Euler-Lagrange equations and high-order diffusion equations.

\section{High-order diffusion and undecimated frame shrinkage corre- spondence}

In this section we consider general frame filter banks and derive the nonlinear diffusion equations associated with them. Recall that for a pair of frame filter banks $\left\{p, q^{(1)}, \cdots, q^{(L)}\right\}$ and $\left\{\widetilde{p}, \widetilde{q}^{(1)}, \cdots, \widetilde{q}^{(L)}\right\}, L_{n}$ and $H_{n}^{(\ell)}$ are the outputs of initial data $\left\{c_{k}\right\}_{k}$ after analysis algorithm $(2.3)$ at p.4, and $u_{k}$ is the shrunk data given by (2.4) with shrinkage functions $S_{\theta_{\ell}}^{\ell}, 1 \leq \ell \leq L$. Observe that if the shrinking operators $S_{\theta_{\ell}}^{\ell}$ in (2.4) are the identity operator (namely, no shrinking process is applied), then $u_{k}=c_{k}$ if and only if this pair of frame filter banks satisfy (2.1). We call $\left\{p, q^{(1)}, \cdots, q^{(L)}\right\}$ and $\left\{\widetilde{p}, \widetilde{q}^{(1)}, \cdots, \widetilde{q}^{(L)}\right\}$ a pair undecimated bi-frame filter banks if they satisfy $(2.1) .\left\{p, q^{(1)}, \cdots, q^{(L)}\right\}$ is called an undecimated tight frame filter bank if it satisfies (2.1) with $\widetilde{p}=p, \widetilde{q}^{(1)}=q^{(1)}, \cdots, \widetilde{q}^{(L)}=q^{(L)}$. In this section we derive high-order nonlinear diffusion equations associated with undecimated frame filter banks. In $\S 3.1$, we obtain a proposition which rewrites $u_{k}$ in a formula which is closely related to a discretized version of some high-order diffusion equations. In $\S 3.2$, we derive the correspondence between nonlinear diffusion equations and undecimated bi-frame filter banks.

\subsection{Undecimated bi-frame shrinkage}

First we have the following lemma.

Lemma 1. Let $\left\{p, q^{(1)}, \cdots, q^{(L)}\right\}$ and $\left\{\widetilde{p}, \widetilde{q}^{(1)}, \cdots, \widetilde{q}^{(L)}\right\}$ be a pair of undecimated bi-frame filter banks, namely they satisfy (2.1). Then

$$
\sum_{m \in \mathbb{Z}} \widetilde{p}_{n} p_{n+j}=4 \delta(j)-\sum_{\ell=1}^{L} \sum_{n \in \mathbb{Z}} \widetilde{q}_{n}^{(\ell)} q_{n+j}^{(\ell)}, j \in \mathbb{Z},
$$

where $\delta(j)$ denotes the Kronecker delta sequence with $\delta(j)=1$ if $j=0$, and $\delta(j)=0$ if $j \neq 0$. 
Proof. Denote $\widetilde{q}^{(0)}(\omega)=\widetilde{p}(\omega), q^{(0)}(\omega)=p(\omega)$. From (2.1), we have

$$
\left(\frac{1}{2}\right)^{2} \sum_{\ell=0}^{L} \sum_{m \in \mathbb{Z}} q_{m}^{(\ell)} e^{i m \omega} \sum_{n \in \mathbb{Z}} \widetilde{q}_{n}^{(\ell)} e^{-i n \omega}=1 .
$$

Using the substitution $m=n+j$, we have

$$
\frac{1}{4} \sum_{\ell=0}^{L} \sum_{n \in \mathbb{Z}} \sum_{j \in \mathbb{Z}} \widetilde{q}_{n}^{(\ell)} q_{n+j}^{(\ell)} e^{i j \omega}=1
$$

which is equivalent to

$$
\sum_{\ell=0}^{L} \sum_{n \in \mathbb{Z}} \widetilde{q}_{n}^{(\ell)} q_{n+j}^{(\ell)}=4 \delta(j), j \in \mathbb{Z}
$$

Thus (3.1) holds.

Proposition 1. Suppose $\left\{p, q^{(1)}, \cdots, q^{(L)}\right\}$ and $\left\{\widetilde{p}, \widetilde{q}^{(1)}, \cdots, \widetilde{q}^{(L)}\right\}$ are a pair of undecimated biframe filter banks, namely they satisfy (2.1). Let $u_{k}$ be the resulting signal given by (2.4) after 1-step frame shrinking of $c_{k}$ with these filter banks. Then

$$
u_{k}=c_{k}+\left.\frac{\sqrt{2}}{4} \sum_{\ell=1}^{L} \sum_{m \in \mathbb{Z}} \widetilde{q}_{m}^{(\ell)}\left(S_{\theta_{\ell}}^{\ell}(x)-x\right)\right|_{x=H_{k-m}^{(\ell)}}, k \in \mathbb{Z},
$$

where $H_{m}^{(\ell)}$ is defined by $(2.3)$.

Proof. By (3.1), we know the first summation in the right hand side of equation (2.4) for $u_{k}$ is

$$
\begin{aligned}
& \frac{1}{2 \sqrt{2}} \sum_{n \in \mathbb{Z}} \widetilde{p}_{n} L_{k-n}=\frac{1}{4} \sum_{n \in \mathbb{Z}} \widetilde{p}_{n} \sum_{m \in \mathbb{Z}} p_{m} c_{m+k-n}=\frac{1}{4} \sum_{n \in \mathbb{Z}} \sum_{j \in \mathbb{Z}} \widetilde{p}_{n} p_{n+j} c_{k+j} \\
& =\sum_{j \in \mathbb{Z}}\left(\delta(j)-\frac{1}{4} \sum_{\ell=1}^{L} \sum_{n \in \mathbb{Z}} \widetilde{q}_{n}^{(\ell)} q_{n+j}^{(\ell)}\right) c_{k+j}=c_{k}-\frac{1}{4} \sum_{\ell=1}^{L} \sum_{j \in \mathbb{Z}} \sum_{n \in \mathbb{Z}} \widetilde{q}_{n}^{(\ell)} q_{n+j}^{(\ell)} c_{k+j} \\
& =c_{k}-\frac{\sqrt{2}}{4} \sum_{\ell=1}^{L} \sum_{n \in \mathbb{Z}} \widetilde{q}_{n}^{(\ell)} \frac{1}{\sqrt{2}} \sum_{j \in \mathbb{Z}} q_{n+j}^{(\ell)} c_{k+j}=c_{k}-\frac{\sqrt{2}}{4} \sum_{\ell=1}^{L} \sum_{n \in \mathbb{Z}} \widetilde{q}_{n}^{(\ell)} H_{k-n}^{(\ell)} .
\end{aligned}
$$

Thus,

$$
\begin{aligned}
u_{k} & =c_{k}-\frac{\sqrt{2}}{4} \sum_{\ell=1}^{L} \sum_{n \in \mathbb{Z}} \widetilde{q}_{n}^{(\ell)} H_{k-n}^{(\ell)}+\frac{1}{\sqrt{2}} \sum_{\ell=1}^{L} \sum_{n \in \mathbb{Z}} \widetilde{q}_{n}^{(\ell)} S_{\theta_{\ell}}^{\ell}\left(H_{k-n}^{(\ell)}\right) \\
& =c_{k}+\left.\frac{\sqrt{2}}{4} \sum_{\ell=1}^{L} \sum_{m \in \mathbb{Z}} \widetilde{q}_{n}^{(\ell)}\left(S_{\theta_{\ell}}^{\ell}(x)-x\right)\right|_{x=H_{k-n}^{(\ell)}},
\end{aligned}
$$

as desired. 


\subsection{High-order nonlinear diffusion equation}

For a (highpass) filter $q(\omega)=\frac{1}{2} \sum_{k \in \mathbb{Z}} q_{k} e^{-i k \omega}$, we say that it has vanishing moment order $J$ if

$$
\sum_{k \in \mathbb{Z}} k^{j} q_{k}=0, \forall j \text { with } 0 \leq j<J .
$$

The vanishing moments of analysis highpass filters imply the annihilation of discrete polynomials in the analysis step or decomposition algorithm, which results in sparse representations of input data.

Denote

$$
C_{J}=\frac{1}{J !} \sum_{k \in \mathbb{Z}} k^{J} q_{k}
$$

Clearly, if $q(\omega)$ does not have vanishing moment order $J+1$, then $C_{J} \neq 0$. Next we have a result which can be found in [37] about using a highpass filter for the approximation of the derivative of a function.

Lemma 2. If an FIR filter $q(\omega)$ has vanishing moment order $J($ not $J+1)$, then for a function $F(x)$ smooth enough,

$$
\begin{aligned}
& \frac{1}{C_{J}} \frac{1}{\varepsilon^{J}} \sum_{k \in \mathbb{Z}} q_{k} F(x+k \varepsilon)=F^{(J)}(x)+o(1), \\
& \frac{1}{C_{J}} \frac{(-1)^{J}}{\varepsilon^{J}} \sum_{k \in \mathbb{Z}} q_{k} F(x-k \varepsilon)=F^{(J)}(x)+o(1),
\end{aligned}
$$

where $C_{J}$ is defined by (3.3).

Proof. Using L'Hospital's Rule repeatedly, we have

$$
\begin{aligned}
& \lim _{\varepsilon \rightarrow 0} \frac{1}{\varepsilon^{J}} \sum_{k \in \mathbb{Z}} q_{k} F(x+k \varepsilon)=\lim _{\varepsilon \rightarrow 0} \frac{1}{\frac{d}{d \varepsilon}\left(\varepsilon^{J}\right)} \frac{d}{d \varepsilon}\left(\sum_{k \in \mathbb{Z}} q_{k} F(x+k \varepsilon)\right) \\
& =\lim _{\varepsilon \rightarrow 0} \frac{1}{J \varepsilon^{J-1}} \sum_{k \in \mathbb{Z}} k q_{k} F^{\prime}(x+k \varepsilon)=\lim _{\varepsilon \rightarrow 0} \frac{1}{J \frac{d}{d \varepsilon}\left(\varepsilon^{J-1}\right)} \frac{d}{d \varepsilon}\left(\sum_{k \in \mathbb{Z}} k q_{k} F^{\prime}(x+k \varepsilon)\right) \\
& =\cdots=\lim _{\varepsilon \rightarrow 0} \frac{1}{J !} \sum_{k \in \mathbb{Z}} k^{J} q_{k} F^{(J)}(x+k \varepsilon)=\frac{1}{J !}\left(\sum_{k \in \mathbb{Z}} k^{J} q_{k}\right) F^{(J)}(x) .
\end{aligned}
$$

Thus we have (3.4). (3.5) follows from (3.4) with $\varepsilon$ replaced by $-\varepsilon$.

Let $\left\{p, q^{(1)}, \cdots, q^{(L)}\right\}$ and $\left\{\widetilde{p}, \widetilde{q}^{(1)}, \cdots, \widetilde{q}^{(L)}\right\}$ be a pair of frame filter banks satisfying (2.1). Assume that $\widetilde{q}^{(\ell)}$ and $q^{(\ell)}$ have vanishing moment orders $\alpha_{\ell}$ (not $\left.\alpha_{\ell}+1\right)$ and $\beta_{\ell}\left(\right.$ not $\left.\beta_{\ell}+1\right)$ respectively. Consider the following nonlinear diffusion equation for $u=(x, t)$ :

$$
u_{t}=\sum_{\ell=1}^{L}(-1)^{1+\alpha_{\ell}} \frac{\partial^{\alpha_{\ell}}}{\partial x^{\alpha_{\ell}}}\left(g_{\ell}\left(\left(\frac{\partial^{\beta_{\ell}} u}{\partial x^{\beta_{\ell}}}\right)^{2}\right) \frac{\partial^{\beta_{\ell}} u}{\partial x^{\beta_{\ell}}}\right),
$$

with $f$ as initial condition:

$$
u(x, 0)=f(x) .
$$


Again, denote $u_{k}^{0}=f(k h)$, and let $u_{k}^{j}$ denote the approximation to the value $u(k h, j \tau)$ of $u(x, t)$ at $(k h, j \tau)$, where $h$ and $\tau$ are the spatial step size and the time step size. For the $\ell$-th term in (3.6), we use following formulas to approximate partial derivatives $\frac{\partial^{\beta} \ell u}{\partial x^{\beta} \ell}$ and $\frac{\partial^{\alpha} \ell}{\partial x^{\alpha} \ell} G(x, t)$, where $G(x, t):=g_{\ell}\left(\left(\frac{\partial^{\beta} \ell u}{\partial x^{\beta} \ell}\right)^{2}\right) \frac{\partial^{\beta_{\ell u}}}{\partial x^{\beta_{\ell}}}$ :

$$
\begin{aligned}
& \frac{\partial^{\beta_{\ell}} u}{\partial x^{\beta_{\ell}}}(k h, j \tau) \approx \frac{1}{C_{\beta_{\ell}}} \frac{1}{h^{\beta_{\ell}}} \sum_{n \in \mathbb{Z}} q_{n}^{(\ell)} u(k h+n h, j \tau) \approx \frac{1}{C_{\beta_{\ell}}} \frac{1}{h^{\beta_{\ell}}} \sum_{n \in \mathbb{Z}} q_{n}^{(\ell)} u_{n+k}^{j}, \\
& \frac{\partial^{\alpha_{\ell}}}{\partial x^{\alpha_{\ell}}} G(k h, j \tau) \approx \frac{(-1)^{\alpha_{\ell}}}{\widetilde{C}_{\alpha_{\ell}}} \frac{1}{h^{\alpha_{\ell}}} \sum_{m \in \mathbb{Z}} \widetilde{q}_{m}^{(\ell)} G(k h-m h, j \tau)
\end{aligned}
$$

where $C_{\beta_{\ell}}$ and $\widetilde{C}_{\alpha_{\ell}}$ are the constants defined by (3.3) with $q^{(\ell)}$ and $\widetilde{q}^{(\ell)}$ respectively. Observe that (3.7) and (3.8) follow from (3.4) and (3.5) respectively with $\varepsilon=h$.

With (3.7) and (3.8), (3.6) can be discretized as

$$
u_{k}^{j+1}=u_{k}^{j}+\tau \sum_{\ell=1}^{L}(-1)^{1+\alpha_{\ell}} \frac{(-1)^{\alpha_{\ell}}}{\widetilde{C}_{\alpha_{\ell}}} \frac{1}{h^{\alpha_{\ell}}} \sum_{m \in \mathbb{Z}} \widetilde{q}_{m}^{(\ell)} g_{\ell}\left(\left(\frac{1}{C_{\beta_{\ell}}} \frac{1}{h^{\beta_{\ell}}} \sum_{n \in \mathbb{Z}} q_{n}^{(\ell)} u_{n+(k-m)}^{j}\right)^{2}\right)\left(\frac{1}{C_{\beta_{\ell}}} \frac{1}{h^{\beta_{\ell}}} \sum_{n \in \mathbb{Z}} q_{n}^{(\ell)} u_{n+(k-m)}^{j}\right) .
$$

In particular, with $c_{k}=u_{k}^{0}$, the above equation for $j=0$ is

$$
u_{k}^{1}=c_{k}-\tau \sum_{\ell=1}^{L} \frac{1}{\widetilde{C}_{\alpha_{\ell}} h^{\alpha_{\ell}}} \sum_{m \in \mathbb{Z}} \widetilde{q}_{m}^{(\ell)} g_{\ell}\left(\left(\frac{\sqrt{2}}{C_{\beta_{\ell}} h^{\beta_{\ell}}} H_{k-m}^{(\ell)}\right)^{2}\right)\left(\frac{\sqrt{2}}{C_{\beta_{\ell}} h^{\beta_{\ell}}} H_{k-m}^{(\ell)}\right),
$$

where $H_{m}^{(\ell)}$ is defined by $(2.3)$.

Comparing (3.2) with (3.9), we have the following result.

Theorem 2. Let $u_{k}$ be the resulting signal in (2.4) after 1-step of frame shrinking of $c_{k}=$ $f(k h), k \in \mathbb{Z}$ with undecimated bi-frame filter banks $\left\{p, q^{(1)}, \cdots, q^{(L)}\right\}$ and $\left\{\widetilde{p}, \widetilde{q}^{(1)}, \cdots, \widetilde{q}^{(L)}\right\}$ and shrinkage functions $S_{\theta_{\ell}}^{\ell}$. Let $u_{k}^{1}$ in $(3.9)$ be the signal after 1-step diffusing defined above for diffusion equation (3.6) with $u_{k}^{0}=f(k h), k \in \mathbb{Z}$ as the initial input. If

$$
S_{\theta_{\ell}}^{\ell}(x)=x\left(1-\frac{4 \tau}{\widetilde{C}_{\alpha_{\ell}} C_{\beta_{\ell}} h^{\alpha_{\ell}+\beta_{\ell}}} g_{\ell}\left(\frac{2 x^{2}}{\left(C_{\beta_{\ell}}\right)^{2} h^{2 \beta_{\ell}}}\right)\right), 1 \leq \ell \leq L,
$$

then $u_{k}=u_{k}^{1}$ for all $k$.

For the tight frame filter bank, $\widetilde{p}=p$ and $\widetilde{q}^{(\ell)}=q^{(\ell)}$. In this case the nonlinear diffusion equation corresponding to the tight frame shrinking is

$$
u_{t}=\sum_{\ell=1}^{L}(-1)^{1+\beta_{\ell}} \frac{\partial^{\beta_{\ell}}}{\partial x^{\beta_{\ell}}}\left(g_{\ell}\left(\left(\frac{\partial^{\beta_{\ell}} u}{\partial x^{\beta_{\ell}}}\right)^{2}\right) \frac{\partial^{\beta_{\ell}} u}{\partial x^{\beta_{\ell}}}\right),
$$

with $f$ as initial condition:

$$
u(x, 0)=f(x) .
$$


For undecimated tight frame filter banks, the formulas used to discretize partial derivatives $\frac{\partial^{\beta} \ell u}{\partial x^{\beta_{\ell}}}$ and $\frac{\partial^{\beta_{\ell}}}{\partial x^{\beta_{\ell}}} G(x, t)$, for $G(x, t):=g_{\ell}\left(\left(\frac{\partial^{\beta_{\ell u}}}{\partial x^{\beta_{\ell}}}\right)^{2}\right) \frac{\partial^{\beta} \ell u}{\partial x^{\beta_{\ell}}}$ are

$$
\begin{aligned}
& \frac{\partial^{\beta_{\ell}} u}{\partial x^{\beta_{\ell}}}(k h, j \tau) \approx \frac{1}{C_{\beta_{\ell}} h^{\beta_{\ell}}} \sum_{n \in \mathbb{Z}} q_{n}^{(\ell)} u_{n+k}^{j}, \\
& \frac{\partial^{\beta_{\ell}}}{\partial x^{\beta_{\ell}}} G(k h, j \tau) \approx \frac{(-1)^{\beta_{\ell}}}{C_{\beta_{\ell}} h^{\beta_{\ell}}} \sum_{m \in \mathbb{Z}} q_{m}^{(\ell)} G(k h-m h, j \tau) .
\end{aligned}
$$

Then $u_{k}^{1}$ after 1-step diffusing is

$$
u_{k}^{1}=c_{k}-\tau \sum_{\ell=1}^{L} \frac{1}{C_{\beta_{\ell}} h^{\beta_{\ell}}} \sum_{m \in \mathbb{Z}} q_{m}^{(\ell)} g_{\ell}\left(\left(\frac{\sqrt{2}}{C_{\beta_{\ell}} h^{\beta_{\ell}}} H_{k-m}^{(\ell)}\right)^{2}\right)\left(\frac{\sqrt{2}}{C_{\beta_{\ell}} h^{\beta_{\ell}}} H_{k-m}^{(\ell)}\right),
$$

where $H_{m}^{(\ell)}$ is defined by (2.3). Comparing (3.2) with (3.12), we have the following result, which is a special case of Theorem 2 .

Theorem 3. Let $u_{k}$ be the resulting signal in (2.4) after 1-step of frame shrinking of $c_{k}=$ $f(k h), k \in \mathbb{Z}$ with an undecimated tight frame filter bank $\left\{p, q^{(1)}, \cdots, q^{(L)}\right\}$ and shrinkage functions $S_{\theta_{\ell}}^{\ell}$. Let $u_{k}^{1}$ in (3.12) be the signal after 1-step diffusing defined above for diffusion equation (3.11) with $u_{k}^{0}=f(k h), k \in \mathbb{Z}$. If

$$
S_{\theta_{\ell}}^{\ell}(x)=x\left(1-\frac{4 \tau}{\left(C_{\beta_{\ell}}\right)^{2} h^{2 \beta_{\ell}}} g_{\ell}\left(\frac{2 x^{2}}{\left(C_{\beta_{\ell}}\right)^{2} h^{2 \beta_{\ell}}}\right)\right), 1 \leq \ell \leq L,
$$

then $u_{k}=u_{k}^{1}$ for all $k$.

Theorem 3 reveals the connection between nonlinear diffusion equations and general undecimated tight frame shrinkages. As in Section 2.3, one can show that the nonlinear diffusion equation (3.11) is related to the Euler-Lagrange equation of a variational functional.

Since bi-frame (tight frame) filter banks are undecimated bi-frame (tight frame) filter banks, all results above hold true for bi-frame (tight frame) filter banks. Next, let us look at Ron-Shen's tight frame filter bank again to illustrate the general theorem.

Example 1. Let $\left\{p, q^{(1)}, q^{(2)}\right\}$ be Ron-Shen's tight frame filter bank defined by (2.5). Then $\beta_{1}=1, C_{\beta_{1}}=-\sqrt{2} ; \beta_{2}=2, C_{\beta_{2}}=-\frac{1}{2}$. Thus $S_{\theta_{1}}^{1}(x)$ and $S_{\theta_{2}}^{2}(x)$ in (3.13) are

$$
\begin{aligned}
& S_{\theta_{1}}^{1}(x)=x-\frac{4 \tau}{\left(C_{\beta_{1}}\right)^{2} h^{2}} g_{1}\left(\frac{2 x^{2}}{\left(C_{\beta_{1}}\right)^{2} h^{2}}\right) x=x-\frac{4 \tau}{(-\sqrt{2})^{2} h^{2}} g_{1}\left(\frac{2 x^{2}}{(-\sqrt{2})^{2} h^{2}}\right) x=x-\frac{2 \tau}{h^{2}} g_{1}\left(\frac{x^{2}}{h^{2}}\right) x, \\
& S_{\theta_{2}}^{2}(x)=x-\frac{4 \tau(-1)^{\beta_{2}}}{\left(C_{\beta_{2}}\right)^{2} h^{4}} g_{2}\left(\frac{2 x^{2}}{\left(C_{\beta_{2}}\right)^{2} h^{4}}\right) x=x-\frac{4 \tau(-1)^{2}}{\left(-\frac{1}{2}\right)^{2} h^{4}} g_{2}\left(\frac{2 x^{2}}{\left(-\frac{1}{2}\right)^{2} h^{4}}\right) x=x-\frac{16 \tau}{h^{4}} g_{2}\left(\frac{8 x^{2}}{h^{4}}\right) x .
\end{aligned}
$$

Therefore relationship (3.13) of the diffusivity and shrinkage functions for this tight frame bank coincides with that in (2.11) with $\theta_{1}=\theta, \theta_{2}=\sigma$. 


\section{More high-order nonlinear diffusion equations}

In this section we construct two sets of tight frame filter banks which result in the 6th- and 8th-order nonlinear diffusion equations. In the following, denote

$$
z=e^{-i \omega}
$$

\subsection{Sixth-order nonlinear diffusion equation}

In this subsection we construct a tight frame filter bank with three highpass filters $q^{(\ell)}, 1 \leq \ell \leq 3$. $q^{(\ell)}$ has vanishing moment order $\ell$, and it is symmetric or antisymmetric around the origin, namely, $q^{(\ell)}(-\omega)=q^{(\ell)}(\omega)$ or $q^{(\ell)}(-\omega)=-q^{(\ell)}(\omega)$. We consider the filters that are supported on $[-2,2]$. That is the coefficients $q_{k}^{(\ell)}=0$ if $|k|>2$.

First let us look at $q^{(3)}$. If it has vanishing moment order 3 , and it is antisymmetric around the origin and supported on $[-2,2]$, then it can be written as

$$
q^{(3)}(\omega)=\frac{1}{2} e_{0}\left(-\frac{1}{2} z^{-2}+z^{-1}-z+\frac{1}{2} z^{2}\right)
$$

for some $e_{0} \in \mathbb{R}$. The formula in Lemma 2 for the 3 rd derivative $L^{(3)}(x)$ of a function $L(x)$ related to such a $q^{(3)}(\omega)$ is

$$
L^{(3)}\left(x_{0}\right)=\frac{1}{\varepsilon^{3}}\left(-L\left(x_{0}-2 \varepsilon\right)+2 L\left(x_{0}-\varepsilon\right)-2 L\left(x_{0}+\varepsilon\right)+L\left(x_{0}+2 \varepsilon\right)\right)+O\left(\varepsilon^{2}\right) .
$$

Next we consider $q^{(1)}(\omega)$ and $q^{(2)}(\omega)$. We choose $q^{(1)}(\omega)$ to be the filter given by

$$
q^{(1)}(\omega)=\frac{1}{2} \frac{c_{0}}{12}\left(z^{-2}-8 z^{-1}+8 z-z^{2}\right),
$$

where $c_{0} \in \mathbb{R}$. The reason for such a choice of $q^{(1)}$ is that the corresponding formula for the derivative $L^{\prime}(x)$ of a function $L(x)$ is the so-called five-point formula (see e.g. [2]):

$$
L^{\prime}\left(x_{0}\right)=\frac{1}{12 \varepsilon}\left(L\left(x_{0}-2 \varepsilon\right)-8 L\left(x_{0}-\varepsilon\right)+8 L\left(x_{0}+\varepsilon\right)-L\left(x_{0}+2 \varepsilon\right)\right)+O\left(\varepsilon^{4}\right) .
$$

For $q^{(2)}(\omega)$, we hope that the corresponding formula for the derivative $L^{\prime \prime}(x)$ is similar to the five-point formula for $L^{\prime}(x)$ :

$$
L^{\prime \prime}\left(x_{0}\right)=\frac{1}{12 \varepsilon^{2}}\left(-L\left(x_{0}-2 \varepsilon\right)+16 L\left(x_{0}-\varepsilon\right)-30 L\left(x_{0}\right)+16 L\left(x_{0}+\varepsilon\right)-L\left(x_{0}+2 \varepsilon\right)\right)+O\left(\varepsilon^{4}\right) .
$$

$q^{(2)}(\omega)$ corresponding to the formula (4.5) is given by

$$
q^{(2)}(\omega)=\frac{1}{2} \frac{d_{0}}{12}\left(-z^{-2}+16 z^{-1}-30+16 z-z^{2}\right),
$$

where $d_{0} \in \mathbb{R}$. Let $p(\omega)$ be the lowpass filter given

$$
p(\omega)=\frac{1}{2}\left(b_{0} z^{-2}+\frac{1}{2} z^{-1}+1-2 b_{0}+\frac{1}{2} z+b_{0} z^{2}\right),
$$


where $b_{0} \in \mathbb{R}$.

For $p, q^{(1)}, q^{(2)}$ and $q^{(3)}$ given by (4.7), (4.3), (4.6) and (4.1), we find that we are unable to choose $b_{0}, c_{0}, d_{0}, e_{0}$ such that $p, q^{(1)}, q^{(2)}$ and $q^{(3)}$ form a tight frame filter bank with the resulting scaling function being in $L^{2}(\mathbb{R})$. Because of this we consider $q^{(2)}(\omega)$ given by

$$
q^{(2)}(\omega)=\frac{1}{2} \frac{d_{0}}{a_{0}-2}\left(-z^{-2}+\left(a_{0}+2\right) z^{-1}-2 a_{0}-2+\left(a_{0}+2\right) z-z^{2}\right),
$$

where $d_{0}, a_{0} \in \mathbb{R}, a_{0} \neq 2$. The corresponding formula for the 2 nd derivative $L^{\prime \prime}(x)$ of a function $L(x)$ is

$$
\begin{aligned}
L^{\prime \prime}\left(x_{0}\right)=\frac{1}{\left(a_{0}-2\right) \varepsilon^{2}}( & -L\left(x_{0}-2 \varepsilon\right)+\left(a_{0}+2\right) L\left(x_{0}-\varepsilon\right)-\left(2 a_{0}+2\right) L\left(x_{0}\right) \\
& \left.+\left(a_{0}+2\right) L\left(x_{0}+\varepsilon\right)-L\left(x_{0}+2 \varepsilon\right)\right)+O\left(\varepsilon^{2}\right) .
\end{aligned}
$$

Then we can choose $a_{0}, b_{0}, c_{0}, d_{0}, e_{0}$ such that the resulting scaling function is in $L^{2}(\mathbb{R})$. More precisely, if

$$
b_{0}=\frac{3 \sqrt{2}-14}{178}, c_{0}=\sqrt{2+16 b_{0}}, d_{0}=\frac{7+104 b_{0}}{6}, e_{0}=\frac{\sqrt{-14-256 b_{0}}}{6}, a_{0}=\frac{233+2314 b_{0}}{21},
$$

then $\phi$ is $W^{1.20414}(\mathbb{R})$ and $p, q^{(1)}, q^{(2)}, q^{(3)}$ form a tight frame filter bank. The corresponding nonlinear diffusion equation is

$$
u_{t}=\frac{\partial}{\partial x}\left(g_{1}\left(\left(\frac{\partial u}{\partial x}\right)^{2}\right) \frac{\partial u}{\partial x}\right)-\frac{\partial^{2}}{\partial x^{2}}\left(g_{2}\left(\left(\frac{\partial^{2} u}{\partial x^{2}}\right)^{2}\right) \frac{\partial^{2} u}{\partial x^{2}}\right)+\frac{\partial^{3}}{\partial x^{3}}\left(g_{3}\left(\left(\frac{\partial^{3} u}{\partial x^{3}}\right)^{2}\right) \frac{\partial^{3} u}{\partial x^{3}}\right) .
$$

In the above paragraph and also in the next subsection, for $s>0, W^{s}(\mathbb{R})$ denotes the Sobolev space which consists of all functions $f$ on $\mathbb{R}$ satisfying $\int_{\mathbb{R}}\left(1+|\omega|^{2}\right)^{s}|\widehat{f}(\omega)|^{2} d \omega<\infty$, where $\widehat{f}(\omega)$ denotes the Fourier transform of $f(x)$. The Sobolev exponent of a compactly supported scaling function $\phi$ can be characterized by the eigenvalues of the transition operator associated with the refinement mask of $\phi$. The reader is referred to $[17,34,19]$ for the characterization, and to [20] for the Matlab routines about calculating the Sobolev smoothness of $\phi$.

For $q^{(1)}, q^{(2)}$ and $q^{(3)}$ given by (4.3), (4.8) and (4.1) respectively, one can calculate directly that their vanishing moment orders $\beta_{j}$ and the corresponding $C_{\beta_{j}}$ defined by (3.3) are

$$
\beta_{1}=1, C_{\beta_{1}}=c_{0} ; \beta_{2}=2, C_{\beta_{2}}=d_{0} ; \beta_{3}=3, C_{\beta_{3}}=e_{0} .
$$

Thus, the relationship between $S_{\theta_{\ell}}^{\ell}$ and $g_{\ell}$ in Theorem 3 is given by

$S_{\theta_{1}}^{1}(x)=x\left(1-\frac{4 \tau}{c_{0}^{2} h^{2}} g_{1}\left(\frac{2 x^{2}}{c_{0}^{2} h^{2}}\right)\right), S_{\theta_{2}}^{2}(x)=x\left(1-\frac{4 \tau}{d_{0}^{2} h^{4}} g_{2}\left(\frac{2 x^{2}}{d_{0}^{2} h^{4}}\right)\right), S_{\theta_{3}}^{3}(x)=x\left(1-\frac{4 \tau}{e_{0}^{2} h^{6}} g_{3}\left(\frac{2 x^{2}}{e_{0}^{2} h^{6}}\right)\right)$,

where $h$ and $\tau$ are the spatial step size and the time step size respectively.

In conclusion, with the relationship in (4.11) for the diffusivity and shrinkage functions, the signal resulted from iterated denoising with $p, q^{(1)}, q^{(2)}$ and $q^{(3)}$ of the tight frame filter bank given by (4.7), (4.3), (4.8) and (4.1) respectively and that resulted from diffusion governed by equation (4.10) with the discretization of the 1st, 2nd and 3rd partial derivatives given by (4.4), (4.9) and (4.2) respectively are the same. 


\subsection{Eighth-order nonlinear diffusion equation}

In this subsection, we construct a tight frame filter bank with four symmetric/antisymmetric highpass filters $q^{(\ell)}, 1 \leq \ell \leq 4$. $q^{(\ell)}$ has vanishing moment order $\ell$, and all the filters constructed are supported on $[-2,2]$. This filter bank results in a 8th-order nonlinear diffusion equation.

Let $p, q^{(1)}, q^{(2)}$ and $q^{(3)}$ be the filters given by (4.7), (4.3), (4.6) and (4.1) respectively. We construct the 4 th highpass $q^{(4)}$ to have vanishing moment order 4 and to be symmetric around the origin. Then $q^{(4)}$ is given by

$$
q^{(4)}(\omega)=\frac{1}{2} f_{0}\left(z^{-2}-4 z^{-1}+6-4 z+z^{2}\right),
$$

where $f_{0} \in \mathbb{R}$. The corresponding formula for the 4 th-order derivative $L^{(4)}$ of a function $L(x)$ is

$$
L^{(4)}\left(x_{0}\right)=\frac{1}{\varepsilon^{4}}\left(L\left(x_{0}-2 \varepsilon\right)-4 L\left(x_{0}-\varepsilon\right)+6 L\left(x_{0}\right)-4 L\left(x_{0}+\varepsilon\right)+L\left(x_{0}+2 \varepsilon\right)\right)+O\left(\varepsilon^{2}\right) .
$$

If we choose

$$
b_{0}=\frac{\sqrt{7057}-95}{192}, c_{0}=\sqrt{2+16 b_{0}}, d_{0}=\frac{\sqrt{7+104 b_{0}}}{4}, e_{0}=\frac{\sqrt{-14-256 b_{0}}}{6}, f_{0}=\frac{589+624 b_{0}}{54},
$$

then $\phi$ is in the Sobolev space $W^{1.19195}(\mathbb{R})$, and $\left\{p, q^{(1)}, q^{(2)}, q^{(3)}\right\}$ is a tight frame filter bank. The corresponding nonlinear diffusion equation is

$$
\begin{aligned}
u_{t}=\frac{\partial}{\partial x} & \left(g_{1}\left(\left(\frac{\partial u}{\partial x}\right)^{2}\right) \frac{\partial u}{\partial x}\right)-\frac{\partial^{2}}{\partial x^{2}}\left(g_{2}\left(\left(\frac{\partial^{2} u}{\partial x^{2}}\right)^{2}\right) \frac{\partial^{2} u}{\partial x^{2}}\right) \\
& +\frac{\partial^{3}}{\partial x^{3}}\left(g_{3}\left(\left(\frac{\partial^{3} u}{\partial x^{3}}\right)^{2}\right) \frac{\partial^{3} u}{\partial x^{3}}\right)-\frac{\partial^{4}}{\partial x^{4}}\left(g_{4}\left(\left(\frac{\partial^{4} u}{\partial x^{4}}\right)^{2}\right) \frac{\partial^{4} u}{\partial x^{4}}\right) .
\end{aligned}
$$

For $q^{(1)}, q^{(2)}, q^{(3)}$ and $q^{(4)}$ given by (4.3), (4.6), (4.1) and (4.12), one can calculate directly that their vanishing moment orders $\beta_{j}$ and the corresponding $C_{\beta_{j}}$ defined by (3.3) are

$$
\beta_{1}=1, C_{\beta_{1}}=c_{0} ; \beta_{2}=2, C_{\beta_{2}}=d_{0} ; \beta_{3}=3, C_{\beta_{3}}=e_{0} ; \beta_{4}=4, C_{\beta_{4}}=f_{0} .
$$

Thus the relationship between $S_{\theta_{\ell}}^{\ell}$ and $g_{\ell}$ in Theorem 3 is given by

$$
\begin{aligned}
& S_{\theta_{1}}^{1}(x)=x\left(1-\frac{4 \tau}{c_{0}^{2} h^{2}} g_{1}\left(\frac{2 x^{2}}{c_{0}^{2} h^{2}}\right)\right), S_{\theta_{2}}^{2}(x)=x\left(1-\frac{4 \tau}{d_{0}^{2} h^{4}} g_{2}\left(\frac{2 x^{2}}{d_{0}^{2} h^{4}}\right)\right), \\
& S_{\theta_{3}}^{3}(x)=x\left(1-\frac{4 \tau}{e_{0}^{2} h^{6}} g_{3}\left(\frac{2 x^{2}}{e_{0}^{2} h^{6}}\right)\right), S_{\theta_{4}}^{4}(x)=x\left(1-\frac{4 \tau}{f_{0}^{2} h^{8}} g_{4}\left(\frac{2 x^{2}}{f_{0}^{2} h^{8}}\right)\right) .
\end{aligned}
$$

With such a relation among the diffusivity and shrinkage functions, the signal resulted from iterated denoising with the tight frame filter bank given by (4.7), (4.3), (4.6), (4.1) and (4.12) and that resulted from diffusion governed by equation (4.14) with the discretization of the 1st to 4th partial derivatives given by (4.4), (4.5), (4.2), (4.13) are the same.

The reader is referred to [12] for a tight frame filter bank consisting of 4 highpass filters $q^{(\ell)}, 1 \leq \ell \leq 4$ with $q^{(\ell)}$ having vanishing moment order $\ell$. Its corresponding scaling function is the $C^{2}$ cubic spline supported on $[-2,2]$; and its first two highpass filters $q^{(1)}, q^{(2)}$ are different from 
these given above (they cannot result in five-point formulas (4.4)(4.5) for the 1st- and 2nd-order derivatives), while up to constants, $q^{(3)}$ and $q^{(4)}$ are the filters given in (4.1) and (4.12).

Remark 1. In this section we construct two sets of tight frame filter banks which result in the 6th- and 8th-order nonlinear diffusion equations. If we consider undecimated tight frame filter banks, then we will have more flexibility for the construction which will result in smoother scaling functions. The details related to such construction are omitted here.

\section{$5 \quad$ Experimental results}

We carried out various experiments of signal denoising based on Ron-Shen's tight frame filter bank with different shrinkage functions. The overall performances of the diffusion-inspired shrinking (except for the one from TV diffusivity) are comparable with hard and soft threshold denoising. Actually, they perform slightly better. Here we provide experimental results with two "toy" signals, denoted as $S_{1}$ and $S_{2}$.

For $S_{1}$, which is shown on the top-left of Fig. 1, five noised signals are generated by adding zero-mean Gaussian noise five times to the original signal $S_{1}$. Each noised signal has signal-tonoise ratio $(\mathrm{SNR})=6$. SNR is defined as

$$
\mathrm{SNR}=20\left(\log _{10}|s-\bar{s}|_{2}-\log _{10}|n|_{2}\right),
$$

where $s$ is the ideal signal and $\bar{s}$ is the mean of $s$, and $n$ is the noise. We apply 1-level RonShen's frame shrinking iteratively 50 times to each noised signal. We provide in Table 1 the SNRs of the denoised signals with different shrinkage functions. The SNR for each case in Table 1 is the average of the SNRs of the denoised signals of the five noised signals mentioned above. When we apply the Perona-Malik (denoted as $\mathrm{P}_{-} \mathrm{M}$ ) diffusivity function, we choose $h=1, \tau=\frac{1}{4}$. We choose $c_{1}=1$ when Perona-Malik diffusivity-based $S_{\theta}^{1}$ given in (2.13) is applied to the first highpass output $H_{n}^{(1)}$ while we set $c_{2}=\frac{1}{8}$ when Perona-Malik diffusivity-based $S_{\sigma}^{2}$ in $(2.13)$ is applied to the second highpass output $H_{n}^{(2)}$. We set $h=1, \tau=\frac{1}{4}$ when Weickert diffusivity-based $S_{\theta}^{1}$ defined by (2.15) is applied to the first highpass output $H_{n}^{(1)}$, while we use $h=1, \tau=\frac{1}{16}$ when Weickert diffusivity-based $S_{\sigma}^{2}$ in (2.16) is applied to the second highpass output $H_{n}^{(2)}$. The parameters $\theta$ and $\sigma$ are selected such that SNRs of the denoised signals are as big as possible. The TV diffusivity-based $S_{\theta}^{1}, S_{\sigma}^{2}$ defined by (2.14) are independent of the parameters. Here we choose a smaller $\tau$ with $\tau=\frac{1}{32}$. From Table 1, we know for $S_{1}$, Perona-Malik diffusivity-inspired and Weickert diffusivity-inspired shrinkages perform slightly better than hard and soft shrinkages.

\begin{tabular}{|l|l|l|l|l|l|l|l|}
\hline Shrinkage & $S_{\theta}^{1}=\mathrm{P}_{-} \mathrm{M}$ & $S_{\theta}^{1}=$ Weickert & $S_{\theta}^{1}=$ Hard & $S_{\theta}^{1}=$ Soft & $S_{\theta}^{1}=$ P_M & $S_{\theta}^{1}=$ Weickert & $S_{\theta}^{1}=\mathrm{TV}$ \\
Method & $S_{\sigma}^{2}=\mathrm{P}_{-} \mathrm{M}$ & $S_{\sigma}^{2}=$ Weickert & $S_{\sigma}^{2}=$ Hard & $S_{\sigma}^{2}=$ Soft & $S_{\sigma}^{2}=$ Weickert & $S_{\sigma}^{2}=$ P_M & $S_{\sigma}^{2}=\mathrm{TV}$ \\
\hline SNR (for $\left.S_{1}\right)$ & 18.0560 & 18.0643 & 17.4083 & 17.8367 & 18.0589 & 18.0539 & 16.2321 \\
\hline SNR (for $S_{2}$ ) & 26.1805 & 25.6973 & 24.4256 & 24.4167 & 26.3698 & 25.6202 & 16.2660 \\
\hline
\end{tabular}

Table 1: Signal denoising results with different shrinkage functions

The second signal $S_{2}$ we consider is shown on the top-left of Fig. 2. Again five noised signals are generated by adding zero-mean Gaussian noise five times to $S_{2}$. In this case each noised signal has SNR=16 and we apply Ron-Shen's frame shrinking iteratively 100 times to each noised signal 

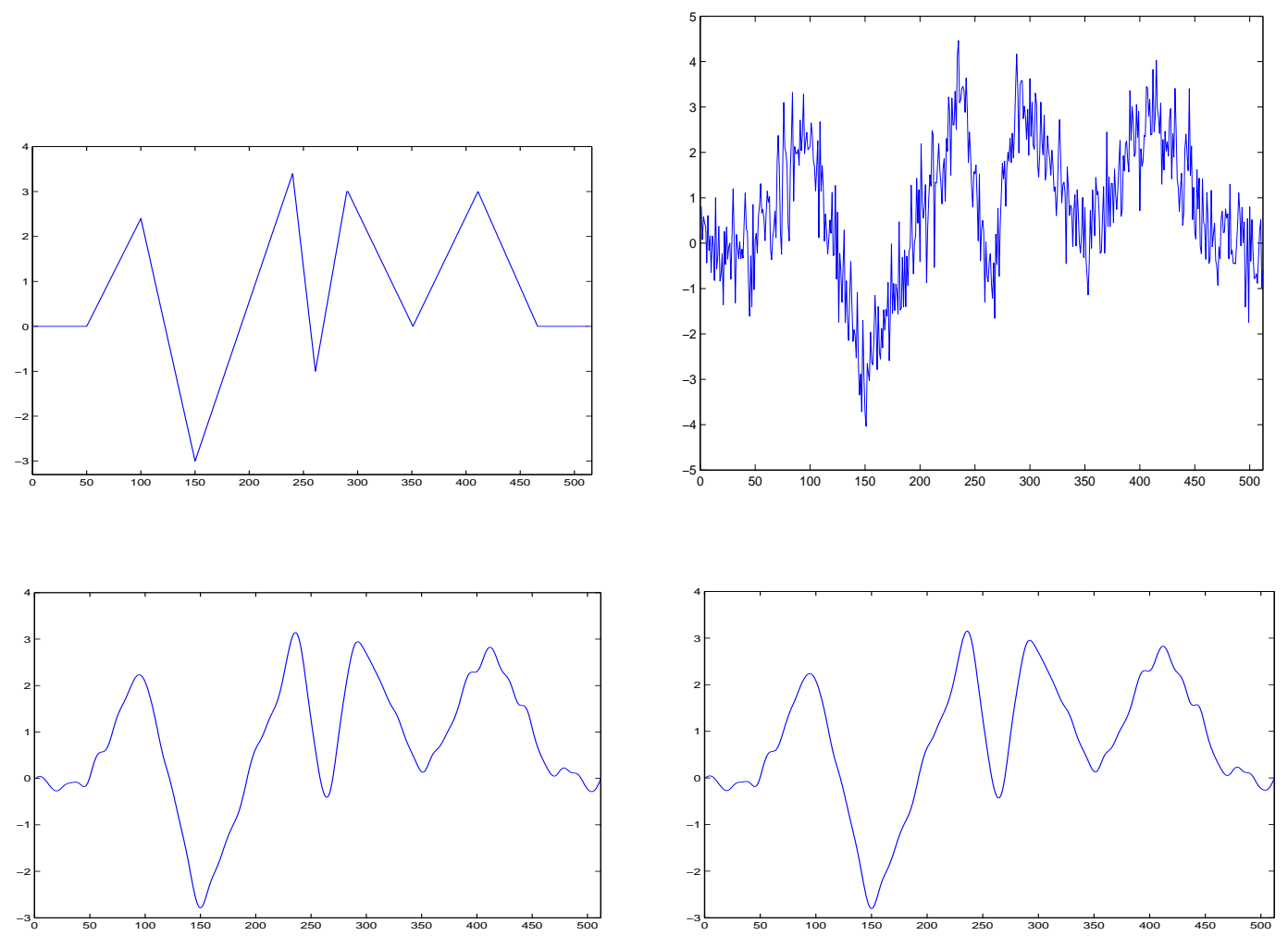

Figure 1: Top-left: Original signal $S_{1}$; Top-right: Noised signal with SNR=6; Bottom-left: Denoised signal with Perona-Malik shrinkage; Bottom-right: Denoised signal with Weickert shrinkage

(still 50 times for TV diffusivity-inspired shrinking). The constant $c_{1}, c_{2}$ and $\tau$ are chosen as above. The SNRs of the denoised signals with different shrinkage functions are provided also in Table 1. Again, for each case, the SNR for $S_{2}$ in Table 1 is the average of the SNRs of the denoised signals of five noised signals. This example also shows that Perona-Malik diffusivity-inspired and Weickert diffusivity-inspired shrinkages perform better than hard and soft shrinkages.

\section{Conclusion and future work}

In this paper we establish the correspondence between frame shrinkage functions and the diffusivity functions of certain high-order nonlinear diffusion equations. We start with the frame shrinkage based on a Ron-Shen's continuous-linear-spline-based tight frame filter bank and obtain a 4thorder nonlinear diffusion equation associated with this filter bank. After that we derive high-order nonlinear diffusion equations associated with general tight frame filter banks. These high-order nonlinear diffusion equations are different from the high-order diffusion equations studied in the literature. In addition, we construct two sets of tight frame filter banks which result in the 6thand 8th-order nonlinear diffusion equations. We also present signal denoising experiments with various shrinkage functions including diffusivity-inspired shrinkage functions.

The study of relationship between the frame shrinkage and diffusion filtering leads to a new type of diffusion equations. The derived relationship is useful to design diffusion-inspired shrinkage functions with competitive performance. On the other hand, the relationship is helpful to design 

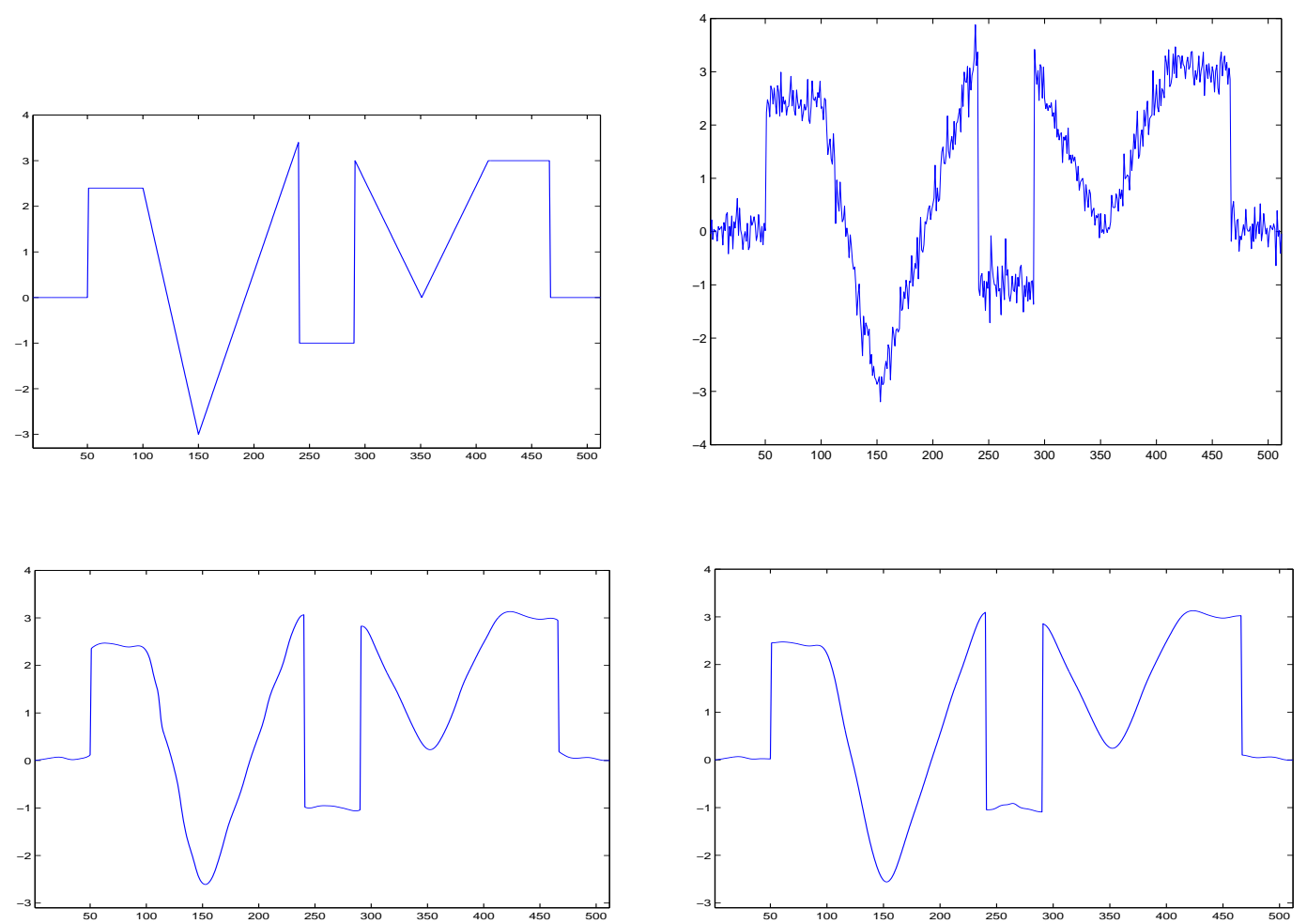

Figure 2: Top-left: Original signal $S_{2}$; Top-right: Noised signal with SNR=16; Bottom-left: Denoised signal with Perona-Malik shrinkage; Bottom-right: Denoised signal with Weickert shrinkage

frame-inspired diffusivity functions.

In this paper we consider frame shrinkage and diffusion filtering correspondence in the 1-D case. Our future work will include the study of the correspondence between 2-D frame shrinkage and diffusion filtering, the design of competitive diffusion-inspired shrinkage functions for image denoising and the construction of tight frame filter banks which result in nonlinear diffusion equations with good performances in image noise removal.

Acknowledgements. The author would like to thank Professor Charles K. Chui for helpful discussions on nonlinear diffusion filtering. Thanks to Dr. Haihui Wang for helpful discussions on the correspondence between wavelet shrinkage and diffusion filtering. The author also thanks two anonymous referees for their valuable comments and suggestions.

\section{References}

[1] D. Barash, Fundamental relationship between bilateral filtering, adaptive smoothing, and the nonlinear diffusion equation, IEEE Trans. Pattern Analysis and Machine Intelligence 24 (2002) 844-847.

[2] R.L. Burden, J.D. Faires, Numerical Analysis (8th Ed.), Thomson Books/Cole, Belmont, CA, 2005.

[3] J.F. Cai, R.H. Chan, L.X. Shen, Z.W. Shen, Restoration of chopped and nodded images by framelets, SIAM J. Sci. Comput. 24 (2008) 1205-1227. 
[4] J.F. Cai, R.H. Chan, Z.W. Shen, A framelet-based image inpainting algorithm, Appl. Comput. Harmonic Anal. 24 (2008) 131-149.

[5] J.F. Cai, S. Osher, Z.W. Shen, Split Bregman methods and frame based image restoration, Multiscale Modeling and Simulation: A SIAM Interdisciplinary Journal 8 (2009) 337-369.

[6] F. Catté, P.-L. Lions, J.-M. Morel, T. Coll, Image selective smoothing and edge detection by nonlinear diffusion, SIAM J. Numer. Anal. 29 (1992) 182-193.

[7] R.H. Chan, T.F. Chan, L.X. Shen, Z.W. Shen, Wavelet algorithms for high-resolution image reconstruction, SIAM Journal Sci. Comput. 24 (2003) 1408-1432.

[8] R.H. Chan, S.D. Riemenschneider, L.X. Shen, Z.W. Shen, Tight frame: An efficient way for high-resolution image reconstruction, Appl. Comput. Harmonic Anal. 17 (2004) 91-115.

[9] A. Chebira, Adaptive Multiresolution Frame Classification of Biomedical Images, Ph.D. Dissertation, Carnegie Mellon University, 2008.

[10] C.K. Chui, J.Z. Wang, PDE models associated with the bilateral filter, Adv. Comput. Math. 31 (2009) 131-156.

[11] R.R. Coifman, D.L. Donoho, Translation-invariant de-noising, in: Wavelets and Statistics, Springer Lecture Notes in Statistics 103, Springer-Verlag, New York, 1994, pp. 125-150.

[12] I. Daubechies, B. Han, A. Ron, Z.W. Shen, Framelets: MRA-based construction of wavelet frames, Appl. Comput. Harmonic Anal. 14 (2003) 1-46.

[13] S. Didas, Denoising and enhancement of digital images-variational methods, integrodifferential equations, and wavelets, Ph.D. Dissertation, Saarland University (2008). http://www.itwm.de/bv/employees/didas/dissertation.pdf.

[14] S. Didas, J. Weickert, B. Burgeth, Properties of higher order nonlinear diffusion filtering, Journal of Mathematical Imaging and Vision 35 (2009) 208-226.

[15] D.L. Donoho, De-noising by soft-thresholding, IEEE Trans. Inform. Theory 41 (1995) 613627.

[16] D.L. Donoho, I.M. Johnstone, Ideal spatial adaptation via wavelet shrinkage, Biometrika 81 (1994) 425-455.

[17] T. Eirola, Sobolev characterization of solutions of dilation equations, SIAM J. Math. Anal. 23 (1992) 1015-1030.

[18] S.D. Gertz, B.G. Bodmann, D. Vela, M. Papadakis, et al, Three-dimensional isotropic wavelets for post-acquisitional extraction of latent images of atherosclerotic plaque components from micro-computed tomography of human coronary arteries, Academic Radiology 17 (2007) 1509-1519.

[19] R.Q. Jia, Q.T. Jiang, Spectral analysis of transition operators and its applications to smoothness analysis of wavelets, SIAM J. Matrix Anal. Appl. 24 (2003) 1071-1109. 
[20] Q.T. Jiang, P. Oswald, Triangular $\sqrt{3}$-subdivision schemes: the regular case, J. Comput. Appl. Math. 156 (2003) 47-75.

[21] M. Lysaker, A. Lundervold, X.-C. Tai, Noise removal using fourth-order partial differential equation with applications to medical magnetic resonance images in space and time, IEEE Trans. Image Process. 12 (2003) 1579-1590.

[22] S. Mallat, A Wavelet Tour of Signal Processing (2nd Ed.), Academic Press, San Diego, 1999.

[23] P. Mrázek, J. Weickert, G. Steidl, Correspondences between wavelet shrinkage and nonlinear diffusion", in: L.D. Griffin and M. Lillholm (Eds.), Scale-Space Methods in Computer Vision, Lecture Notes in Computer Science vol. 2695, Springer, Berlin, 2003, pp. 101-116.

[24] P. Mrázek, J. Weickert, G. Steidl, Diffusion-inspired shrinkage functions and stability results for wavelet denoising, International Journal of Computer Vision 64 (2005) 171-186.

[25] M. Papadakis, B.G. Bodmann, S.K. Alexander, D. Vela, et al, Texture based tissue characterization for high-resolution CT-scans of coronary arteries, Comm. in Numer. Methods in Engineering 25 (2009) 597-613.

[26] P. Perona, J. Malik, Scale space and edge detection using anisotropic diffusion, IEEE Trans. Pattern Analysis and Machine Intelligence 12 (1990) 629-639.

[27] A. Ron, Z.W. Shen, Affine systems in $L_{2}\left(\mathbb{R}^{d}\right)$ : the analysis of the analysis operators, J. Funct. Anal. 148 (1997) 408-447.

[28] A. Ron, Z.W. Shen, Affine systems in $L_{2}\left(\mathbb{R}^{d}\right)$ II: Dual systems, J. Fourier Anal. Appl. 3 (1997) 617-637.

[29] L. Rudin, S. Osher, E. Fatemi, Nonlinear total variation based noise removal algorithms, Physica D 60 (1992) 259-268.

[30] L.X. Shen, M. Papadakis, I.A. Kakadiaris, I.Konstantinidis, I. Kouri, D. Hoffman, Image denoising using a tight frame, IEEE Trans. Image Proc. 15 (2006) 1254-1263.

[31] G. Steidl, J. Weickert, T. Brox, P. Mrázek, M. Welk, On the equivalence of soft wavelet shrinkage, total variation diffusion, total variation regularization, and SIDEs, SIAM J. Numer. Anal. 42 (2004) 686-713.

[32] G. Strang, Introduction to Applied Math, Wellesley-Cambridge Press, Wellesley, MA, 1986.

[33] G. Strang, T. Nguyen, Wavelets and Filter Banks, Wellesley Cambridge Press, Wellesley, 1996.

[34] L. Villemoes, Energy moments in time and frequency for two-scale difference equation solutions and wavelets, SIAM J. Math. Anal. 23 (1992) 1519-1543.

[35] G.W. Wei, Generalized Perona-Malik equation for image restoration, IEEE Signal Process. Lett. 6 (1999) 165-167.

[36] J. Weickert, Anisotropic Diffusion in Image Processing, B.G. Teubner, Stuttgart, 1998. 
[37] J. Weickert, G. Steidl, P. Mrázek, M. Welk, T. Brox, Diffusion filters and wavelets: What can they learn from each other?, in: N. Paragios, Y. Chen, O. Faugeras (Eds.), Handbook of Mathematical Models in Computer Vision, Springer, New York, 2006, pp. 3-16.

[38] Y.-L. You, M. Kaveh, Image enhancement using fourth order partial differential equations, in: 32nd Asilomar Conf. Signals, Systems, Computers, vol. 2, 1998, pp. 1677-1681.

[39] Y.-L. You, M. Kaveh, Fourth-order partial differential equations for noise removal, IEEE Trans. Image Process. 9 (2000) 1723-1730. 